\title{
Erratum: Local structural excitations in model glass systems under applied load [Phys. Rev. B 93, 144202 (2016)]
}

S. Swayamjyoti, J. F. Löffler, and P. M. Derlet

(Received 13 November 2018; published 4 December 2018)

DOI: 10.1103/PhysRevB.98.219904

A conversion factor error was discovered in our previously published work. The ( $x$-axis) stress scale of Figs. 1(b) and 2(b) are too large by a factor of 100 , and the ( $x$-axis) activation volume scale of Fig. 3(b) is too small by a factor of 100 . This results in all activation volumes being too small by a factor of 100. Apart from these figure corrections, the following corrections to the text are necessary:

(1) In the last paragraph of Sec. III B, the activation volume must be changed from $0.005 \sigma_{11}^{3}$ to $0.5 \sigma_{11}^{3}$.

(2) In the last paragraph of Sec. IV, the sentence "The activation volumes obtained in Sec. III B represent only a small fraction of an atomic volume, which is considerably smaller than what is normally seen in experiment." must be changed to "The activation volumes obtained in Sec. III B are comparable to an atomic volume, which is somewhat smaller than what is normally seen in experiment."

These corrections do not affect the other primary conclusions of the paper. 